\title{
V-shape noncoronary sinus remodeling in ascending aortic aneurysm and aortic root ectasia
}

\author{
John A. Elefteriades, MD, ${ }^{a}$ Sven Peterss, MD, ${ }^{a}$ Nariman Nezami, MD, ${ }^{b}$ Gina Gluck, MD, ${ }^{b}$ Wei Sun, PhD, \\ Maryann Tranquilli, RN, ${ }^{a}$ and Bulat A. Ziganshin, $\mathrm{MD}^{\mathrm{a}, \mathrm{d}}$
}

\section{ABSTRACT}

Objective: The study objective was to describe our initial experience performing a V-shaped single sinus remodeling procedure in patients with ascending aortic aneurysm and moderate aortic root ectasia.

Methods: Twelve consecutive patients underwent supracoronary ascending aortic replacement with $\mathrm{V}$-shaped noncoronary sinus remodeling (median age, 63 years [range, 56-77]; 10 patients [83\%] were male). All patients had an ascending aortic aneurysm (median diameter $48 \mathrm{~mm}$ [range, 42-53]) and aortic root ectasia (median root diameter, $43 \mathrm{~mm}$ [range, 38-49.7 mm]). A deep V-shaped (triangular) portion of the noncoronary sinus was excised, and the wall was directly reapproximated in 2 layers, 1 everting mattress suture layer followed by a running over-andover layer.

Results: No technical complication due to root remodeling was observed. All patients survived the initial hospitalization. Only 1 patient required reexploration for bleeding, unrelated to the $\mathrm{V}$-shaped repair. On postoperative computed tomography, every patient showed reduction in maximal aortic root diameter and cross-sectional area. Mean aortic root diameter was reduced from $4.30 \mathrm{~cm}$ (range, 3.82-4.97) to $3.81 \mathrm{~cm}$ (range, 3.58-3.96) $(P<.0006)$. Mean aortic root cross-sectional area was reduced from $1452 \mathrm{~mm}^{2}$ (range, 1327-1615) to $1180 \mathrm{~mm}^{2}$ (range, 961-1328) $(P<.0002)$. Mean wall tension decreased postoperatively by $12 \%$.

Conclusions: The V-shaped resection of the noncoronary sinus is a viable option for patients with moderate aortic root enlargement. This technique reduces aortic root diameter, cross-sectional area, and wall tension. We offer this technique as another option in the surgeon's armamentarium. (J Thorac Cardiovasc Surg 2017;154:72-6)

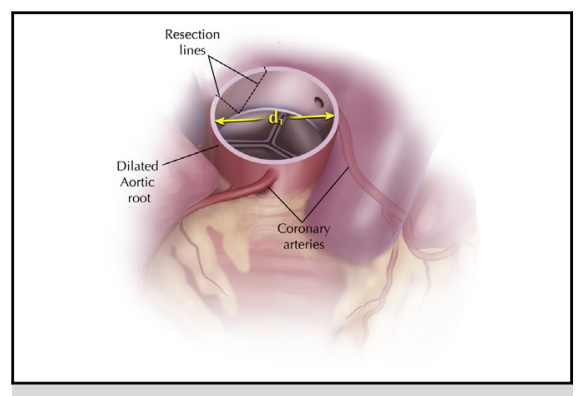

V-shaped noncoronary sinus resection procedure for remodeling of aortic root.

\section{Central Message}

The V-shaped resection of the noncoronary sinus is a viable option for patients with moderate aortic root enlargement.

\section{Perspective}

$\mathrm{V}$-shaped resection of the noncoronary sinus is a simple, quick, reliable supplement to ascending aortic replacement for patients with moderate aortic root enlargement. This technique reduces aortic root diameter, crosssectional area, and wall tension. This approach is useful in elderly patients and those requiring extensive surgery for primary pathologies outside the aortic root.

See Editorial Commentary page 77.
The aortic valve cusps, the annulus, and the sinuses of Valsalva work as 1 functional unit. Restoring or remodeling such a unit adequately is beneficial for functional long-term

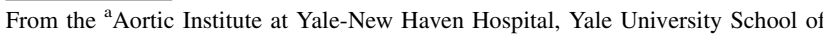
Medicine; ${ }^{\mathrm{b}}$ Department of Radiology and Biomedical Imaging, Yale University School of Medicine, New Haven, Conn; ${ }^{\mathrm{c}}$ The Wallace H. Coulter Department of Biomedical Engineering, Georgia Institute of Technology and Emory University, Atlanta, Ga; and ${ }^{\mathrm{d} D e p a r t m e n t ~ o f ~ S u r g i c a l ~ D i s e a s e s ~ \# 2, ~ K a z a n ~ S t a t e ~ M e d i c a l ~ U n i-~}$ versity, Kazan, Russia.

S.P. is supported by a research fellowship (PE 2206/1-1) of the Deutsche Forschungsgemeinschaft, Bonn, Germany.

Received for publication Oct 19, 2016; revisions received Nov 13, 2016; accepted for publication Nov 28, 2016; available ahead of print Jan 28, 2017.

Address for reprints: John A. Elefteriades, MD, Aortic Institute at Yale-New Haven Hospital, Yale University School of Medicine, Clinic Building CB317, 789 Howard Ave, New Haven, CT 06510 (E-mail: john.elefteriades@yale.edu).

$0022-5223 / \$ 36.00$

Copyright (c) 2016 by The American Association for Thoracic Surgery

http://dx.doi.org/10.1016/j.jtcvs.2016.11.060
}

prognosis. ${ }^{1}$ Ascending aortic aneurysms often include the sinotubular junction (STJ) and extend into the root portion of the aorta, but most commonly affect the noncoronary or right coronary sinus. ${ }^{2}$ Such pathologies (ascending aneurysm with root ectasia) often result in aortic insufficiency. Full aortic root replacement will eradicate all the pathology of the valve, root, and ascending aorta. However, this may represent excessive surgical intervention for an elderly or infirm patient. Valve-sparing aortic root replacement is another option (often accompanied by valve repair);

Scanning this QR code will take you to a video for the article. 


\section{Abbreviation and Acronym}

$\mathrm{STJ}=$ sinotubular junction

however, the same proviso applies regarding the magnitude of operation for the old or infirm. Urbanski and colleagues ${ }^{3}$ has described a more limited approach, with resection of 1 or more sinuses, which are replaced by a shield-shaped patch.

We have accumulated initial experience with a simpler and more expedient option, especially suited to elderly patients, in whom extensive aortic root surgery for sinus ectasia may not be warranted. These are patients generally undergoing operation primarily for ascending aneurysm (body of the ascending aorta), with only mild to moderate enlargement of the aortic root, in the range of 4 to $5 \mathrm{~cm}$. For younger patients and for those with severe root enlargement greater than $5 \mathrm{~cm}$, we use full aortic root replacement. We occasionally apply the V-shaped aortic root remodeling in somewhat younger patients who require extensive additional surgery (eg, additional full aortic arch replacement).

This article describes our initial experience performing a straightforward V-shaped singular sinus remodeling procedure in patients with ascending aortic aneurysm and moderate aortic root ectasia. The V-shaped resection is performed in the noncoronary sinus, the most commonly affected among the 3 sinuses. We largely confine application of this technique to cases in which the aortic valve is simultaneously replaced, usually by a biological prosthesis in this age group. We hesitate to perform this procedure without concomitant aortic valve replacement, because sinus anatomy is changed and aortic insufficiency may be induced, although we did perform this occasionally with preservation of the native valve. Induction of aortic insufficiency is not an issue with concomitant aortic valve replacement. Of course, concomitant aortic valve replacement is a common requirement in the elderly age group because of degeneration or calcification of the valve leaflets.

\section{MATERIALS AND METHODS \\ Surgical Technique}

The patient is prepped and draped in a standard fashion, and a median sternotomy is performed. Cannulation sites are chosen according to the surgeon's preference. After initiating cardiac arrest, the aorta is circularly opened just above the STJ, and, if applicable, the aortic valve is replaced. For remodeling the root, a deep V-shaped or triangular portion of the noncoronary sinus is excised and the wall is directly reapproximated in 2 layers, 1 everting mattress suture layer (4-0 pledgeted Ethibond; Ethicon, Somerville, NJ) and then a running over-and-over layer (4-0 Prolene). The edges of the remnant portions of the noncoronary sinus come together easily, without tension, even after a wide resection.

The proximal anastomosis of the ascending aortic replacement is performed to the smaller cuff (equal to the new STJ) of the aortic root by running suture. We often add a dab of BioGlue (CryoLife, Kennesaw, $\mathrm{Ga})$ at the site where the V-shaped resection comes together with the proximal anastomosis of the main ascending aortic graft, for added security. We routinely reinforce the posterior wall of nearly all aortic anastomoses with multiple interrupted sutures (4-0 Ethibond; Ethicon) for added security. After performing the distal aortic anastomosis, the patient is weaned from cardiopulmonary bypass, and the procedure is completed in the usual fashion (See Figure 1 and Video 1).

\section{RESULTS}

\section{Patient Population and Operative Data}

Between March 2013 and May 2016, 12 consecutive patients underwent supracoronary ascending aortic replacement with V-shaped noncoronary sinus remodeling. Their median age was 63 years (range, 56-77), body mass

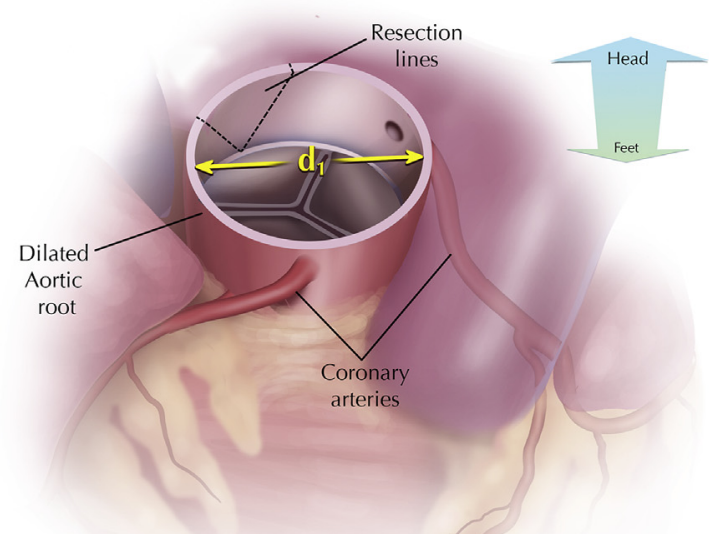

A
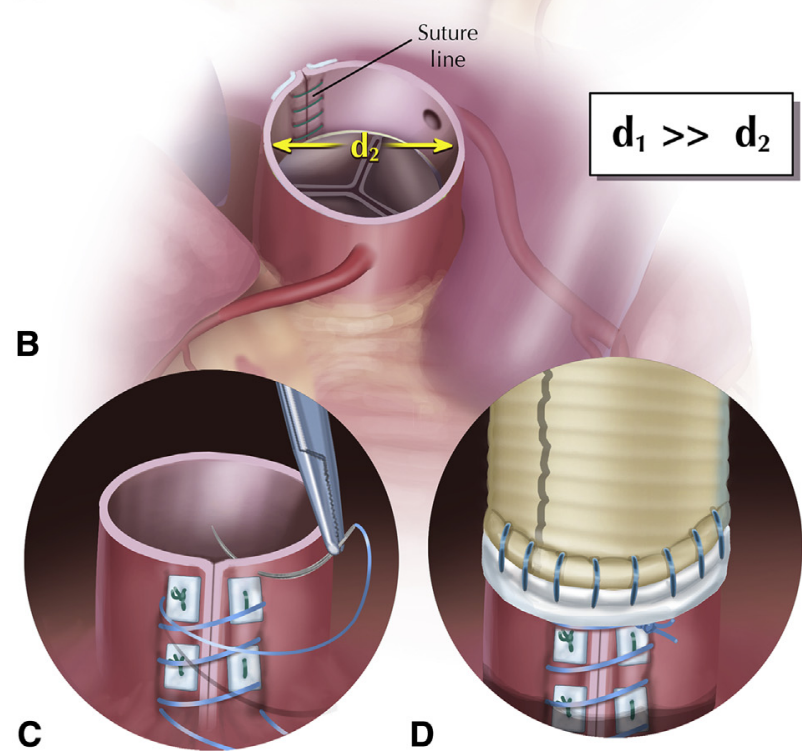

FIGURE 1. Artist's rendition of V-shaped noncoronary sinus resection procedure for remodeling of aortic root. A, Line of resection indicated. B, Reapproximation after resection. C, Details of first everting layer of pledgeted sutures and second running layer. D, End-to-end attachment of ascending graft to remodeled aortic root. 


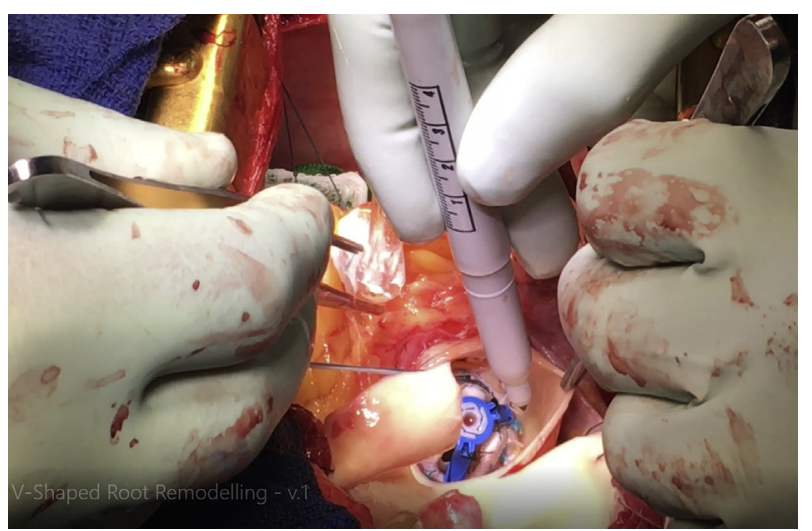

VIDEO 1. A video illustration of the V-shaped noncoronary sinus resection procedure for aortic root remodeling. Video available at: http:// www.jtcvsonline.org/article/S0022-5223(16)31685-3/addons.

index was $28.2(21.9-39.1)$, and $10(83 \%)$ were male. All patients had ascending aortic aneurysms (median diameter of $48 \mathrm{~mm}$ [range, 42-53] and aortic root ectasia [sizes given later]). The aortic valve was severely diseased in 9 patients ( $75 \%$; 2 via stenosis, 7 via insufficiency) and showed mild regurgitation in 3 patients $(25 \%)$. Bicuspid morphology was found in 3 patients $(25 \%)$. Comorbidities included coronary artery disease in 2 patients $(17 \%)$, chronic obstructive pulmonary disease in 4 patients $(33 \%)$, and arterial hypertension and dyslipidemia in 8 patients each $(67 \%)$. Left ventricular ejection fraction was $64 \%$ (range, 40-70), and creatinine level was 0.85 (range, 0.7-1.0).

All patients underwent supracoronary ascending aortic replacement, 4 patients $(33 \%)$ underwent hemiarch replacement, and 2 patients $(17 \%)$ underwent total arch replacement. The aortic valve was replaced in 10 patients $(83 \%)$ and spared in 2 patients $(17 \%$, both with only preoperative mild valve insufficiency and central jet). Concomitant coronary artery bypass grafting was required in 1 patient $(8.3 \%)$.

Cardiopulmonary bypass and crossclamp times were 153.5 minutes (range, 131-202) and 107.5 minutes (69-136), respectively. Hypothermic circulatory arrest was required in 6 patients $\left(50 \% ; 28\right.$ minutes; $\left.19.5^{\circ} \mathrm{C}\right)$.

\section{Operative Outcome}

No technical complication due to root remodeling was observed, and the aortic valve achieved full competency when preserved $(n=2)$. One patient $(8.3 \%)$ experienced bleeding requiring reexploration (distal aortic site), and 5 patients $(42 \%)$ developed postoperative atrial fibrillation. Otherwise, no other postoperative morbidities were found. Median length of stay in the intensive care unit and in hospital were 4 days (range, 2-5) and 6 days (range, 5-8), respectively. Step-down beds were difficult to obtain during this period of time, and patients often stayed in the intensive care unit for lack of beds for the majority of their hospital stay.

\section{Remodeling Outcome}

Preoperative and postoperative aortic root diameters and cross-sectional areas were compared by an independent radiologist unfamiliar with the patients. These are listed in Table 1. Corresponding contrast computed tomography scans of good quality were available for 10 of the 12 patients. Every patient showed reduction in maximal aortic root diameter and cross-sectional area. Mean aortic root diameter was reduced from $4.30 \mathrm{~cm}$ (range, 3.82-4.97) to $3.81 \mathrm{~cm}$ (range, 3.58-3.96) $(P<.0006)$. Mean aortic root cross-sectional area was reduced from $1452 \mathrm{~mm}^{2}$ (range, $1327-1615)$ to $1180 \mathrm{~mm}^{2}$ (range, $\left.961-1328\right)(P<.0002)$. The simplified formula for Laplace's law $\mathrm{T}=\mathrm{P} \times \mathrm{D}$ shows that mean wall tension (Table 2) decreased by $12 \%$. We used the percent change in diameter to represent the change in wall tension, because tension is proportional to diameter.

\section{DISCUSSION}

The presented surgical technique represents a technically simple and effective option to address moderate sinus dilatation by remodeling the noncoronary sinus. This technique accomplishes significant reduction in transverse diameter and cross-sectional area of the aortic root, with attendant reduction in tension in the aortic wall.

In this initial experience, the V-shaped remodeling was accomplished safely. Only 1 patient required reexploration for bleeding, unrelated to the V-shaped repair.

We believe that this V-shaped technique is appropriate for elderly patients with moderate dilatation of the aortic root segment who require aortic valve replacement for stenosis

TABLE 1. Dimensional parameters, comparing preoperative and postoperative computed tomography scans

\begin{tabular}{lccccc}
\hline & \multicolumn{2}{c}{ Preoperative } & & \multicolumn{2}{c}{ Postoperative } \\
\cline { 2 - 3 } \cline { 5 - 6 } $\begin{array}{c}\text { Patient } \\
\text { no. }\end{array}$ & $\begin{array}{c}\text { Root } \\
\text { diameter }(\mathbf{c m})\end{array}$ & $\begin{array}{c}\text { Root area } \\
\left(\mathbf{m m}^{2}\right)\end{array}$ & $\begin{array}{c}\text { Root } \\
\text { diameter }(\mathbf{c m})\end{array}$ & $\begin{array}{c}\text { Root area } \\
\left(\mathbf{m m}^{2}\right)\end{array}$ \\
\hline 1 & 4.71 & 1597 & 3.96 & 1094 \\
2 & 4.37 & 1521 & & 3.96 & 1328 \\
3 & 4.35 & 1521 & & 3.90 & 1260 \\
4 & 4.16 & 1529 & & 3.96 & 1381 \\
5 & 3.82 & 1229 & & 3.75 & 961 \\
6 & 4.97 & 1505 & & 3.47 & 1017 \\
7 & 4.57 & 1615 & & 3.93 & 1271 \\
8 & 4.30 & 1452 & & 3.94 & 1228 \\
9 & 4.02 & 1327 & & NA & NA \\
10 & 4.16 & 1405 & & 3.58 & 1182 \\
11 & $\mathrm{NA}$ & $\mathrm{NA}$ & $\mathrm{NA}$ & $\mathrm{NA}$ \\
12 & 3.92 & 1272 & & 3.61 & 1081 \\
Mean & $4.30^{*}$ & $1452 \dagger$ & $3.81^{*}$ & $1180 \dagger$ \\
\hline
\end{tabular}

$N A$, Not available (because of noncontrast scan). $* P<.0006 . \dagger P<.0002$. 
TABLE 2. Reduction in wall tension

\begin{tabular}{lc}
\hline Patient no. & Reduction in aortic wall tension (\%) \\
\hline 1 & 16 \\
2 & 10 \\
3 & 11 \\
4 & 5 \\
5 & 2 \\
6 & 31 \\
7 & 14 \\
8 & 9 \\
9 & $\mathrm{NA}$ \\
10 & 14 \\
11 & $\mathrm{NA}$ \\
12 & 8 \\
Mean & $12 \%$ \\
\hline
\end{tabular}

NA, Not available.

or insufficiency. It also may be considered as a simple option for addressing moderate dilatation of the aortic root in patients requiring extensive surgery for other segments of the aorta or for other concomitant cardiac issues (eg, aortic arch replacement, coronary artery bypass, mitral valve surgery). Of note, we do not recommend this technique for patients with Marfan's disease or other connective tissue syndromes, because the tissue weakness is too severe for any procedure short of full aortic root replacement. However, such patients usually would present with maximal dilatation at the root segment, so the root would de facto be the primary focus of surgery.

Westaby and colleagues ${ }^{2}$ and Urbanski and colleagues ${ }^{3}$ have taken an approach of resecting the noncoronary sinus (and other sinuses, when needed). However, both Westaby and colleagues and Urbanski and colleagues use a shield-shaped patch or graft extension to replace the resected noncoronary sinus. Our technique differs in that we approximate the tissues primarily after V-shaped excision, thus accomplishing a reduction in the diameter of the aortic root and reducing the wall stress in that area. Our technique also is suitable for individuals with extensive calcification of the aortic wall around the coronary ostia or severe calcification of the proximal right or left coronary arteries; root replacement may be especially hazardous in such circumstances.

Westaby and colleagues ${ }^{2}$ and Urbanski and colleagues ${ }^{3}$ also noted that the noncoronary sinus is the most commonly and earliest affected by dilatation. This observation is consistent with the concept that branch vessels provide support and reinforcement to the arterial wall. It has been shown ${ }^{4,5}$ that excess collagen fibers reinforce areas of arterial bifurcation, like biological "rebar." Reinforcement from collagen surrounding the right and left coronary arteries may underlie the relative protection of the corresponding sinuses from dilatation.
V-shaped resection has the potential to distort the noncoronary sinus, resulting in prolapse of the noncoronary leaflet and aortic insufficiency. For this reason, we prefer to apply the $\mathrm{V}$-shaped resection when the aortic valve is being replaced, in which case the biological or mechanical prosthetic valve is immune to such distortion. When preserving the valve, the technique of Ugur and colleagues ${ }^{6}$ is preferable; this technique replaces the dilated noncoronary sinus with a tongue-like extension of the main aortic graft, which is sutured into the bed of the resected noncoronary sinus.

We do not recommend the V-shaped technique for aortas greater than $5 \mathrm{~cm}$ in diameter, for which we recommend a formal root replacement type procedure. It is possible that our patients may have done well without the V-shaped resection, but we are wary of leaving behind an aortic dimension greater than $4 \mathrm{~cm}$, for fear of the potential for late dilatory consequences. In the current era of 3-dimensional aortic printing from computed tomography scans, examining printed models may facilitate and enhance resection strategy for the noncoronary sinus.

Why not perform a full aortic root replacement in everyone? We believe strongly in aortic root replacement for severe root pathology. The senior author has personally performed more than 500 aortic root replacements. However, we believe that full root replacement may be more than needed when the major aortic pathology is located elsewhere and, especially, when the patient is elderly or the operation is extremely extensive because of multiple pathologies outside of the aortic root zone. Also, replacing the coronary buttons onto a graft adds a small but important additional risk. In contrast to supracoronary ascending replacement, which does not increase surgical risk when performed concomitantly, ${ }^{8}$ aortic root replacement carries additional technical complexity and, thus, increases the operative risk slightly. ${ }^{7,9}$

In a recent study, ${ }^{10}$ we examined the annual growth rate in an untouched aortic root after supracoronary aortic replacement. We found slow growth of the nonresected root $(0.41 \mathrm{~mm} / \mathrm{y})$ and no instance of dissection in the nonresected root segment. The projected time to reaching a diameter for surgical resection (nominally $5.5 \mathrm{~cm}$ ) was more than 25 years. In the case of V-shaped noncoronary resection, the root is made substantially smaller, so even more indolent behavior of the root can be expected than in our recent study of root sparing without V-shaped resection. Furthermore, it is likely that extensive scarring around the V-shaped resection site will discourage dilatation or free rupture.

\section{Study Limitations}

We have reported a favorable but limited experience with the technique of $\mathrm{V}$-shaped resection of the noncoronary sinus. More experience and a longer follow-up are required 
to determine the appropriate potential role of this technique in the surgeon's armamentarium.

\section{CONCLUSIONS}

We report our initial experience with a simple, quick, reliable V-shaped resection of the noncoronary sinus as a supplement to ascending aortic replacement and aortic valve replacement for patients with moderate aortic root enlargement. This technique reduces aortic root diameter, cross-sectional area, and wall tension. We have found this approach useful in elderly patients and those requiring extensive surgery for primary pathologies outside the aortic root. We believe that this V-shaped resection technique offers another alternative surgical technique for the moderately dilated aortic root, between the full aortic root replacement (which may be more than needed) and leaving the root alone entirely (which may be less than needed). We offer this technique as another option in the surgeon's armamentarium.

\section{Conflict of Interest Statement}

Authors have nothing to disclose with regard to commercial support.

\section{References}

1. Reardon MJ, Walkes JC. Invited commentary. Ann Thorac Surg. 2005;80: $843-4$.
2. Westaby S, Saito S, Anastasiadis K, Moorjani N, Jin XY. Aortic root remodeling in atheromatous aneurysms: the role of selected sinus repair. Eur J Cardiothorac Surg. 2002;21:459-64.

3. Urbanski PP, Zhan X, Hijazi H, Zacher M, Diegeler A. Valve-sparing aortic root repair without down-sizing of the annulus. J Thorac Cardiovasc Surg. 2012;143: 294-302.

4. Kwon GP, Schroeder JL, Amar MJ, Remaley AT, Balaban RS. Contribution of macromolecular structure to the retention of low-density lipoprotein at arterial branch points. Circulation. 2008;117:2919-27.

5. Finlay HM, Whittaker P, Canham PB. Collagen organization in the branching region of human brain arteries. Stroke. 1998;29:1595-601.

6. Ugur M, Schaff HV, Suri RM, Dearani JA, Joyce LD, Greason KL, et al. Late outcome of noncoronary sinus replacement in patients with bicuspid aortic valves and aortopathy. Ann Thorac Surg. 2014;97: 1242-6.

7. Mok SC, Ma WG, Mansour A, Charilaou P, Chou AS, Peterss S. Twenty-five year outcomes following composite graft aortic root replacement. J Card Surg. Dec 14, 2016 [Epub ahead of print].

8. Peterss S, Charilaou P, Dumfarth J, Li Y, Bhandari R, Tranquilli M, et al. Aortic valve disease with ascending aortic aneurysm: impact of concomitant root sparing (supracoronary) aortic replacement in non-syndromic patients. J Thorac Cardiovasc Surg. 2016;152:791-8.e1.

9. Svensson LG, Pillai ST, Rajeswaran J, Desai MY, Griffin B, Grimm R, et al. Long-term survival, valve durability, and reoperation for 4 aortic root procedures combined with ascending aorta replacement. J Thorac Cardiovasc Surg. 2016; 151:764-74.e764.

10. Peterss S, Bhandari R, Rizzo JA, Fang H, Kuzmik GA, Ziganshin BA, et al. The aortic root: natural history after root-sparing ascending replacement in nonsyndromic aneurysmal patients. Ann Thorac Surg. Sep 19, 2016 [Epub ahead of print].

Key Words: aortic aneurysm, aortic root remodeling, aortic root sparing, sinus of Valsalva 